\title{
ENGAGEMENT NO TRABALHO: UMA DISCUSSÃO TEÓRICA
}

\author{
Paulo Cesar Porto-Martins ${ }^{\text {Ie II; }}$ Pedro Guilherme Basso-Machado ; \\ Ana Maria Teresa Benevides-Pereira, II III, « \\ ${ }^{I}$ Universidad Autónoma de Madrid, Madrid, Espanha; \\ II Pontifícia Universidade Católica do Paraná, Curitiba, PR, Brasil; \\ ${ }^{I I I}$ Universidade Estadual de Maringá, Maringá, PR, Brasil
}

\section{RESUMO}

O engagement no trabalho é considerado como um estado cognitivo positivo de realização, relacionado com o mundo laboral, que se caracteriza por três dimensões: vigor, dedicação e absorção. Este é formado por um componente comportamental-energético (vigor), um componente emocional (dedicação) e um componente cognitivo (absorção). O objetivo deste estudo é apresentar uma revisão de publicações cientificas internacionais acerca do constructo. $O$ estudo do engagement no trabalho vem como consequência de inúmeras pesquisas realizadas sobre a sindrome de burnout, corroborando com as premissas da psicologia positiva. Conclui-se que existe deficiência de publicações neste constructo, principalmente em se tratando do contexto brasileiro.

Palavras-chave: engagement; UWES; trabalho; saúde laboral; burnout.

\section{WORK ENGAGEMENT: A THEORIC DISCUSSION}

\begin{abstract}
Work engagement is considered as a positive cognitive state, of realization, related to the labour world, characterized by three dimensions: vigor, dedication and absorption. This construct is composed by a energetic-behavioral component (vigor), a emotional component (dedication), and a cognitive component (absorption). The objective of this study is to present a revision of the scientific international publications about the theme. The study of work engagement comes as a consequence of a numerous researches about the burnout syndrome, corroborating with the positive psychology premises. It's concludes that there is a deficiency of publications about this construct, mainly in the Brazilian context.
\end{abstract}

Keywords: engagement; UWES; work; occupational health; burnout.

\footnotetext{
^ Endereço para correspondência: Universidade Estadual de Maringá, Pró Reitoria de Pesquisa. Av. Colombo, 5790, Bloco F67, Sala 09 - Campus Universitário - 87020-900 - Maringa, PR - Brasil. E-mail:paulocpmar@yahoo.com.br,pgbmachado@yahoo.com.br, anamariabenevides@hotmail.com.
} 


\section{Psicologia Positiva: aspectos históricos}

A psicologia priorizou em sua história um enfoque no estudo dos problemas, sintomas e doenças de pessoas, ou seja, os aspectos negativos foram mais estudados do que os positivos (BAKKER et al., 2008). Isto é exemplificado pelo fato de existirem, aproximadamente, 12 vezes mais artigos sobre estresse, do que sobre bem-estar (SALANOVA; SCHAUFELI, 2009), paradigma este denominado modelo médico tradicional (PENA et al., 2011).

O foco nos aspectos psicológicos positivos pode ser evidenciado desde os anos 1950, a partir de autores como Maslow e Rogers, que contemplavam os recursos e aspectos positivos do funcionamento humano enfatizando seus subsídios para a personalidade (CARROBLES; BENEVIDES-PEREIRA, 2009; SALANOVA; SCHAUFELI, 2009). O autor Martin Seligman, posteriormente, denominou esses aspectos de psicologia positiva, também na direção de priorizar o desenvolvimento ótimo do ser humano, com o objetivo de catalisar uma mudança no foco da psicologia, não apenas enquanto reparação de processos nosológicos, mas também nos aspectos salutares (BAKKER; POCINHO; PERESTRELO, 2011; BAKKER; RODRÍGUEZ-MUÑOZ; DERKS, 2012; LEITER et al., 2011; POCINHO; PERESTRELO, 2011; SALANOVA; SCHAUFELI, 2009; SELIGMAN; CSIKSZENTMIHALYI, 2000).

De acordo com Salanova e Schaufeli (2009, p. 74), a psicologia positiva se difundiu em 1998, quando a mesma foi eleita como tema no mandato de Seligman como presidente da APA (Associação Americana de Psicologia). Ainda de acordo com esses autores, o termo foi originado em 1954 com Maslow na obra "Motivação e Personalidade", em que há um capítulo empregando essa denominação.

Dentro dessa tendência, estudos como Bakker et al. (2007); Bakker et al. (2008); Bakker e Leiter (2010); Carvalho et al. (2006); González-Romá et al. (2006); Kahn (1990); Llorens et al. (2007); Maslach e Leiter (2008); Salanova et. Al. (2000); Salanova e Schaufeli (2009); Schaufeli et al. (2002); Schaufeli e Bakker $(2003,2004)$, entre outros, relatam que este movimento culminou na elaboração do constructo chamado work engagement, como consequência de inúmeras pesquisas realizadas sobre aspectos negativos da saúde laboral, como a síndrome de burnout (BAKKER; DEMEROUTI; XANTHOPOULU, 2012; KUBOTA et al., 2011; KUBOTA et al., 2012; LEITER et al., 2011; MASLACH; SCHAUFELI; LEITER, 2001; MORENO-JIMÉNEZ et al., 2010; POCINHO; PERESTRELO, 2011; SHIMAZU; SCHAUFELI, 2009). Assim sendo, o work engagement é considerado um conceito psicológico recente e prometedor, com relevância tanto para o âmbito dos negócios como para o âmbito acadêmico (MASLACH; LEITER, 2008; POCINHO; PERESTRELO, 2011; SCHAUFELI, 2012).

De acordo com Benevides-Pereira (2001, 2002); Gil-Monte (2005); GilMonte e Peiró (1997); Gil-Monte e Moreno-Jiménez (2007); González-Romá et al. (2006); Leiter e Maslach (1988); Maslach e Goldberg (1998); Maslach, Schaufeli e Leiter (2001); Maslach e Leiter (2008), pode-se entender burnout como consequência de um estado prolongado e crônico de estresse, que ocorre 
quando os métodos de enfrentamento foram insuficientes ou falharam. É uma síndrome relacionada exclusivamente com as condições de trabalho, sendo uma resposta a um estado prolongado e crônico de estresse laboral, caracterizada por: esgotamento, desumanização (ou cinismo) e reduzida realização pessoal no trabalho. Tanto a síndrome de burnout quanto o engagement no trabalho são constructos relacionados com o mundo do trabalho e, de forma geral, se relacionam negativamente entre si.

Segundo Salanova e Schaufeli (2009), no final dos anos 1990 a palavra "Engagement do empregado" (e não "no trabalho") era utilizada pela Organização Gallup Buckingham e Coffman. Estes autores também afirmam que a tradução para outras línguas é complicada, uma vez que até o momento não se encontrou um termo que abranja a natureza do conceito sem cair em repetições, simplismos ou erros. Em convergência com este pensamento será adotada a terminologia inglesa engagement, com a traduação do termo work para trabalho, conforme tradução do manual do Utrecth Work Engagement Scale para a língua portuguesa do Brasil, realizado pelo GEPEB - Grupo de Pesquisas Sobre Estresse e Burnout (SCHAUFELI; BAKKER, 2009).

Com relação aos estudos empíricos do engagement no trabalho, estes são recentes e o primeiro autor que o conceituou academicamente foi Willian A. Kahn, em 1990, no artigo intitulado Psychological conditions of personal engagement and disengagement at work (SALANOVA; SCHAUFELI, 2009; SCHAUFELI, 2012). No mesmo artigo, Kahn também referenciou o conceito oposto, o disengagement (ausência de engagement no trabalho) (KAHN, 1990).

No começo da década de 1990, era possível compreender o constructo engagement no trabalho como "aproveitamento dos membros da organização de seus próprios papéis de trabalho: no engagement, as pessoas utilizam e se expressam a si mesmas física, cognitiva, emocional e mentalmente durante o desenvolvimento de seus papéis" (KAHN, 1990, p. 694).

\section{ENGAGEMENT - FATORES RELACIONADOS E CARACTERÍSTICAS}

Há diversos conceitos que se correlacionam positivamente com o engagement no trabalho, tais como: compromisso organizacional (BAKKER; LEITER, 2010; SALANOVA et al., 2000; POCINHO; PERESTRELO, 2011); conduta extrafunções; iniciativa pessoal; qualidade de serviço; autoeficácia; dependência ao trabalho - workaholism (BAKKER; LEITER, 2010); resiliência (CARVALHO et al., 2006); recursos pessoais; auto eficácia e processos de motivação (SALANOVA; SCHAUFELI, 2009); recursos e demandas laborais (LEITER et al., 2011; MORENO-JIMÉNEZ et al., 2010; SCHAUFELI; BAKKER; VAN RHENEN, 2009; SCHAUFELI; TARIS, 2013; TETRICK; HAIMANN, 2013); participação no trabalho (POCINHO; PERESTRELO, 2011). Ainda, corroborando com essas associações, os autores Torp et al. (2012) afirmam que o constructo se associa com elevadas demandas psicológicas associadas ao trabalho, assim como alto controle e apoio social. 
O engagement no trabalho também pode ser caracterizado por energia, envolvimento e eficácia profissional (MASLACH; LEITER, 2008), que seriam os opostos diretos das três dimensões do burnout. Nesta perspectiva, o constructo é avaliado pelo padrão oposto dos escores das três dimensões do burnout (mensurado) pelo Maslach Burnout Inventory - MBI (MASLACH; JACKSON; LEITER, 1996). Dessa maneira, considera-se engagement no trabalho quando na presença de resultados baixos em esgotamento emocional e cinismo, concomitantemente com resultados altos em eficácia profissional, conforme indicam Maslach et al. (2001), o que não foi sido respaldado por outros autores (BAKKER et al., 2008; SCHAUFELI et al., 2002).

Buscando na literatura (BAKKER et al. 2007; BAKKER et al., 2008; LLORENS et al. 2007; SALANOVA et al. 2000; SALANOVA; SCHAUFELI, 2009; SCHAUFELI et al., 2002; SCHAUFELI; BAKKER, 2004), o conceito de engagement no trabalho é entendido como:

-um constructo motivacional positivo;

-sempre relacionado com o trabalho;

-implicado em sentimento de realização (fulfillment);

-caracterizado por vigor, dedicação e absorção;

-envolvido em um estado positivo da mente (cognição);

-persistente no tempo;

-de natureza motivacional e social;

-associado a um estado afetivo emocional;

-caracterizado por um estado psicológico.

\section{ENGAGEMENT NO TRABALHO : CONCEITO E DIMENSÕES}

Oengagement no trabalhoé composto por um componente comportamentalenergético (vigor), um componente emocional (dedicação) e um componente cognitivo (absorção) (SALANOVA; SCHAUFELI, 2009).

Com relação à avaliação do engagment no trabalho, o Utrecht Work Engagement Scale (UWES) é o instrumento mais utilizado (BAKKER et al., 2008; BAKKER; LEITER, 2010; SALANOVA; SCHAUFELI, 2009; OUWENELL; le BLANC; SCHAUFELI, 2013). Este inventário é constituído de 17 itens elaborados por SchaufelieBakker. Há também uma versão reduzida de nove itens (SALANOVA; SCHAUFELI, 2009; SCHAUFELI; BAKKER, 2003; SCHAUFELI; BAKKER; SALANOVA, 2006). A versão brasileira, segundo Machado e Porto-Martins (2013), já encontra-se traduzida e adaptada por Benevides-Pereira e Porto-Martins, versão que apresentou qualidades psicométricas adequadas em estudo empírico realizado por Machado e Benevides-Pereira (2012). 
De acordo com Acosta, Salanova e Llorens (2011); Bakker et al. (2008); Bakker e Leiter (2010); Bakker et al., (2012); Kubota et al., (2011); Kubota et al., (2012); Pocinho; Perestrelo, 2011; Salanova et al. (2000); Salanova e Schaufeli (2009); Schaufeli et al. (2002); Schaufeli (2012); Torrente et al. (2102), este instrumento é constituído por três dimensões básicas. São elas:

-Vigor, que se caracteriza por:

-altos níveis de energia;

-persistência;

-desejo de esforçar-se no trabalho;

-resiliência mental, considerando-se as atividades laborais.

-Dedicação, que se caracteriza por:

-estar plenamente concentrado (involved) na realização do trabalho;

-inspiração;

-orgulho;

-desafio;

-objetivo;

-significado;

-entusiasmo.

-Absorção, que se caracteriza por:

-estar plenamente concentrado e feliz na realização do trabalho;

-sensação de que o tempo passa "voando";

-dificuldade de desligar do trabalho.

Segundo os autores Bakker et al. (2008); Bakker; Leiter (2010); Maslach; Leiter (2008); Salanova; Schaufeli (2009), alguns dos fatores predisponentes ao desenvolvimento do engagement no trabalho são:

-apoio social;

-desempenho no trabalho;

-recursos pessoais: como autoeficácia e autoestima, capital psicológico positivo, crenças, tipo de enfrentamento utilizado, otimismo;

-recursos e demandas organizacionais;

-resiliência.

A promoção de um desses fatores pode gerar o incremento dos demais, ou seja, eles se relacionam reforçando-se mutuamente e ajudando a desenvolver os demais de uma forma crescente (SALANOVA; BAKKER; LLORENS, 2006; 
SALANOVA; SCHAUFELI, 2009). Estes autores também apontam que as crenças de eficácia se relacionam com a presença de emoções positivas, que, por sua vez, predizem os níveis de engagement no trabalho em relação ao futuro.

Os autores Bakker et al. (2007) apontam que o conceito é preditivo de bom desempenho laboral e satisfação do cliente, assim como a resiliência e os recursos ocupacionais, podem facilitar o engagement no trabalho. Este, por sua vez, também pode ser modulador dos efeitos dos recursos organizacionais sobre a realização do trabalho, o bem- estar e a qualidade de vida em geral (LLORENS et al. (2007).

Pode-se compreender "recursos laborais/organizacionais" como aqueles aspectos físicos, sociais e organizacionais que podem: reduzir as demandas, custos físicos e fisiológicos associados, ser funcionais em alcançar os objetivos laborais e estimular o crescimento pessoal, a aprendizagem e o desenvolvimento (BAKKER; LEITER, 2010). Ainda segundo os mesmos autores, recursos pessoais são autoavaliações positivas que são associadas à resiliência e se referem à habilidade para controlar e gerar impacto sobre seus ambientes de forma exitosa. Também se compreende recursos pessoais como aquelas características positivas de pessoas que têm a capacidade de reduzir ou amortecer o potencial do impacto negativo das demandas, se relaciona mutuamente com os recursos laborais (SALANOVA; SCHAUFELI, 2009). Vale mencionar que esses recursos estão conectados diretamente com a percepção de um indivíduo frente às contingências que o rodeiam, "não são as circunstâncias como tal, senão a forma em que as pessoas as vêem, que as fazem sentir-se felizes ou não." (SALANOVA; SCHAUFELI, 2009, p. 176).

\section{ENGAGEMENT NO TRABALHO E ORGANIZAÇÕES}

No que tange ao ambiente organizacional, não existe um conjunto de benefícios preestabelecidos, recursos ou técnicas nas organizações que funcionem de forma igual em distintos contextos. As boas práticas para o desenvolvimento de recursos humanos, desde as organizações saudáveis, são específicas para cada contexto organizacional (CHIAVENATO, 2008, 2009; SALANOVA; SCHAUFELI, 2009).

A promoção do engagement no trabalho, considerada de uma maneira ampla, que modula os efeitos diretos dos recursos organizacionais sobre o desempenho, o bem-estar e a qualidade de vida em geral (LLORENS et al., 2007) pode ser considerado como um estado positivo que possibilita e facilita o uso dos recursos (SALANOVA; SCHAUFELI, 2009).

Em revisão realizada por Bakker et al. (2008), estes sugerem que a promoção do engagement no trabalho beneficiará não somente os indivíduos, mas também irá gerar vantagem competitiva para as organizações (BAKKER et al., 2008; BAKKER; LEITER, 2010; SALANOVA; SCHÁUFELI, 2009). Confirmando esse raciocínio verificou-se que "vários estudos indicaram que o engagement no trabalho tem consequências positivas nos níveis individual e organizacional" (BAKKER et al., 2007, p. 274). 
O engagement no trabalho é tanto individual, como coletivo e social, estando intimamente conectado com o desenvolvimento organizacional, mediante relações recíprocas que geram consequências positivas para ambos, tanto para o trabalhador quanto para a organização, com resultados organizacionais positivos, levando à redução dos negativos (SALANOVA; SCHAUFELI, 2009). A popularidade do termo nas organizações confirma a dimensão prática do conceito (BAKKER; LEITER, 2010).

De acordo com Salanova e Schaufeli (2009), algumas das estratégias possíveis para melhorar o engagement no trabalho em nível organizacional são:

-socialização e avaliação dos colaboradores;

-seleção de pessoal;

-contrato psicológico;

-auditorias;

-oficinas;

-incremento de recursos laborais;

-procedimentos do trabalho;

-modificações laborais;

-liderança, liderança situacional (transformacional);

-gestão da diversidade;

-relações interpessoais.

\section{EMPREgAdOS ENGAGED}

Os empregados engaged são aqueles com altos níveis de engagement no trabalho (neste texto, não será traduzido o termo engaged pelos mesmos motivos expostos anteriormente referente ao termo engagement) e que, geralmente, possuem: emoções positivas; melhor saúde física e psicológica; criam seus próprios recursos, trabalham duro (vigor); são imersos em suas atividades (dedicação); e se sentem de forma plena e altamente concentrados em seus trabalhos (absorção), (BAKKER et al., 2008; BAKKER; LEITER, 2010).

Outra característica é que esses trabalhadores transferem seu engagement no trabalho para outros: com sua forma de ser, influenciam seus colegas a se comportarem e se sentirem de forma similar (BAKKER et al., 2008; SALANOVA; SCHAUFELI, 2009). Estudos evidenciam que estes aparentam ter melhor desempenho e seus clientes se mostram mais satisfeitos (BAKKER et al., 2008; BAKKER; LEITER, 2010). Esses empregados têm uma conexão energética e efetiva com suas atividades laborais e se sentem capazes de administrar as demandas provenientes de seu mundo profissional (SCHAUFELI et al., 2002). 
Dentro da óptica organizacional, esses trabalhadores são avaliados positivamente por supervisores e colegas, têm êxito em seus postos de trabalho - sendo menos tendenciosos a mostrar condutas contra produtivas - têm menor rotatividade e absenteísmo laboral - sendo menos inclinados a desenvolver burnout - mostram mais condutas positivas além de seus papéis, assim como melhor desempenho e produtividade (SALANOVA; SCHAUFELI, 2009).

Mesmo que se tenha diferentes visões de engagement no trabalho, a maioria dos pesquisadores estão de acordo que empregados engaged têm altos níveis de energia e se identificam fortemente com suas atividades ocupacionais (BAKKER et al., 2008; BAKKER; LEITER, 2010).

Pelo fato do engagement no trabalho ser ainda um conceito recente, abibliografia disponível ainda é restrita. Em pesquisa na internet realizada por Salanova e Schaufeli (2009) utilizando como palavras chave "engagement do empregado" e "engagement no trabalho" encontraram duas mil publicações científicas, sendo que ainda menos estudos (cem artigos) tendo estas palavras no título ou no resumo.

\section{ENGAGEMENT NO TRABALHO - EstUdOS}

Entre artigos que contemplam engagement no trabalho, observou-se que grande parte deles procurou diferenciá-lo de demais constructos, assim como identificar seus principais antecedentes e consequentes (BAKKER et al., 2008). Outros aspectos encontrados na literatura recente são a necessidade de desenvolvimento de programas de intervenção para promoção do work engagement; a investigação de indicadores psicofisiológicos presentes no processo de engagement; a realização de pesquisas longitudinais relacionando o termo com a saúde; o estudo das variações diárias nos elementos nucleares de energia e dedicação para melhor compreender suas dinâmicas; a tentativa de compreender o manejo individual dos níveis de engagement no trabalho; a realização de pesquisas em nível interpessoais visando a entender as relações de contingência, assim como os potenciais efeitos negativos que possam existir em consequência do constructo (BAKKER; LEITER, 2010; SALANOVA; SCHAUFELI, 2009).

Quanto à associação entre engagement no trabalho e à síndrome de burnout, verificou-se que esta ocorre por fatores distintos, independentes e negativamente correlacionados (BAKKER et al., 2008). Os estudos sobre esses dois constructos levaram a considerar que estes possuem estruturas e, portanto, formas de avaliação distintas, pois "diferentemente de Maslach e Leiter (1997), nós não cremos que engagement é adequadamente medido pelo perfil opositor dos resultados do MBI." (SCHAUFELI et al., 2002, p.75):

Por último, algumas pesquisas propuseram que "vigor" seja associado ao esgotamento emocional como polos opostos de um mesmo continuum, compondo uma mesma dimensão chamada "Energia", com uma extremidade positiva e outra negativa. A mesma analogia é válida para a relação da dedicação com o cinismo (despersonalização), constituindo uma dimensão chamada "Identificação" (BAKKER; LEITER, 2010; GONZÁLEZ-ROMÁ et al., 2006; LEITER et al., 
2011; POCINHO; PERESTRELO, 2011; SALANOVA et al., 2000; SCHAUFELI et al., 2002; SCHAUFELI; BAKKER, 2004). Nesta perspectiva, o engagement no trabalho é compreendido como uma experiência subjetiva contendo duas dimensões: uma que seria a energia e outra que seria a identificação, que, para alguns, também é chamada de envolvimento (BAKKER; LEITER, 2010). Dessa maneira, segundo Bakker et al. (2008); Salanova e Schaufeli (2009); Schaufeli et al. (2002), o burnout se caracterizaria por uma combinação de pouca energia (esgotamento) e pouca identificação (cinismo), enquanto o engagement no trabalho é assinalado como um padrão oposto: alta energia (vigor) e elevada identificação (dedicação). Assim mesmo, o que se propõe é que os constructos de burnout e engagement no trabalho sejam avaliados por distintos instrumentos de medida (SCHAUFELI et al., 2002).

Independentemente da perspectiva, deve-se considerar que o engagement no trabalho terá uma contribuição maior, haja vista que é concebido como um constructo único e distinto de outros constructos psicológicos tradicionais (BAKKER et al. 2008; BAKKER; LEITER, 2010; SALANOVA; SCHAUFELI, 2009).

Em suma, o desenvolvimento histórico, assim como a correlação entre burnout e engagement no trabalho, podem ser sintetizados no esquema apresentado na Figura I.

Figura 1 - Esquema burnout X Engagement no trabalho

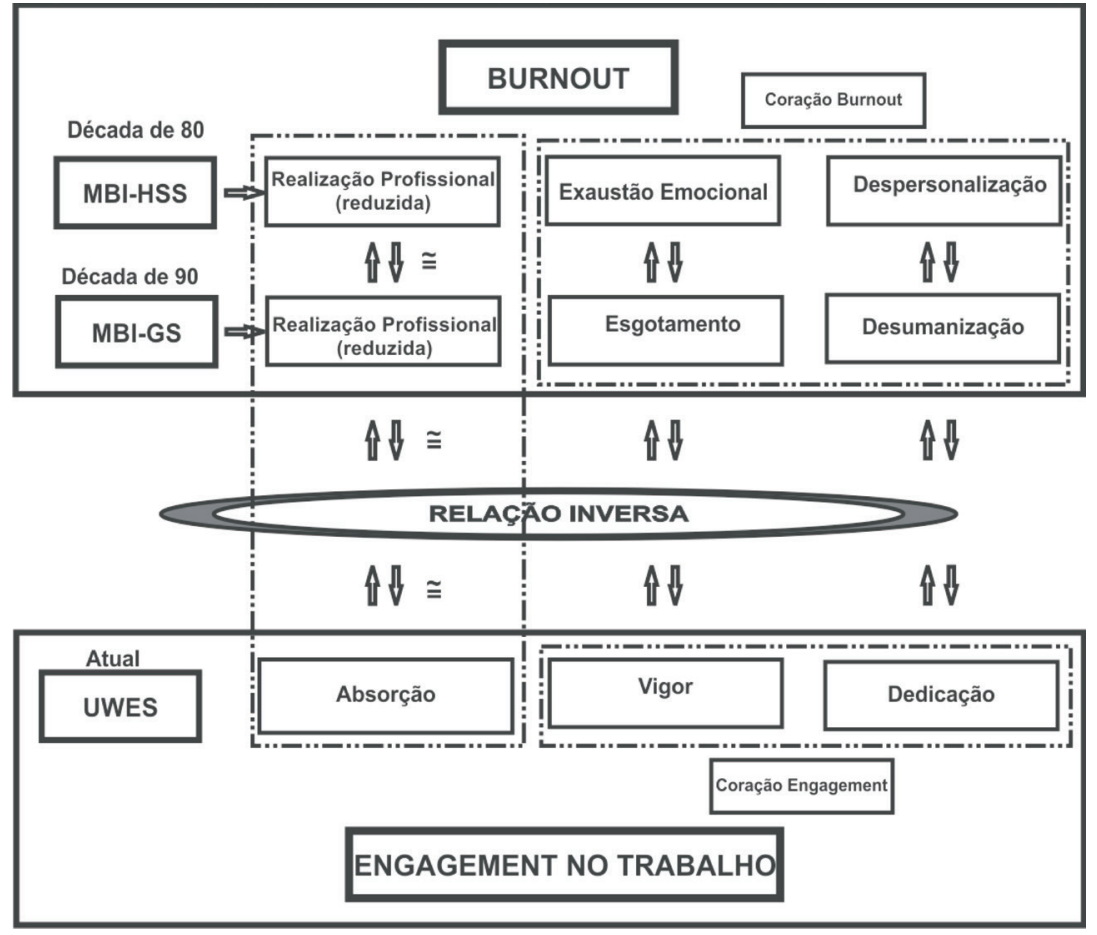

Legenda: Coração burnout = composto pelas dimensões "exaustão emocional" e "despersonalização" do MBI-HSS (Maslach Burnout Inventory-Human Services Survey) ou "esgotamento" e "desumanização" do Maslach Burnout Inventory General Survey - MBI-GS (LEITER et al., 2011; MORENO-JIMÉNEZ et al., 2010). 
Coração Engagement = composto pelas dimensões "vigor" e "dedicação" avaliadas pelo UWES (ACOSTA, et al., 2011; BAKKER; LEITER, 2010; MORENO-JIMÉNEZ et al., 2010).

Assim, deve-se levar em conta, ainda, que o engagement no trabalho terá uma contribuição maior, se for compreendido como um constructo único e distinto de outros constructos psicológicos tradicionais (BAKKER et al. 2008; BAKKER; LEITER, 2010; SALANOVA; SCHAUFELI, 2009). Desenvolver estudos sobre o engagement no trabalho mostra-se um desafio importante, pois implementa uma visão mais positiva em relação ao indivíduo, com foco prioritariamente na saúde.

\section{ENGAGEMENT NO TRABALHO - BRASIL}

Em relação ao Brasil, a carência de publicações sobre o constructo é incipiente, denotando a necessidade de se ampliarem as pesquisas científicas no Brasil referente ao engagement no trabalho.

Por fim, realizando uma pesquisa em distintas bases de dados, como MedLine, SciELO, Lilacs e Ibecs assim como nos principais eventos científicos do Brasil e o site do professor Schaufeli, de forma cronológica, encontraram-se as seguintes publicações:

- Agnst R.; Benevides-Pereira, A. M. T.; Porto-Martins, P. C. (2009). Tradução e Adaptação do manual do UWES para português-BR.

- Machado, P. G. B. (2010). Engagement e burnout em motoristas e cobradores de ônibus. (Primeira publicação sobre Engagement no trabalho no Brasil).

- Porto-Martins, P. C.; Machado, P. G. B. (2010). Engagement no trabalho em motoristas e cobradores de ônibus.

- Porto-Martins, P. C.; Machado, P. G. B. (2010). Engagement en el trabajo. (Resenha).

- Pocinho, M.; Perestrelo, C. X. (2011). Um ensaio sobre burnout, engagement e estratégias de coping na profissão docente. (Trabalho teórico, publicado por pesquisadores portugueses em periódico brasileiro).

- Porto-Martins, P. C.; Amorim, C. (2011) Estresse e engagement no trabalho docente.

- Machado, P. G. B.; Benevides-Pereira, A. M. T. (2012). Engagement y burnout en profesionales de transporte público urbano. (Trabalho de Investigación para la obtención del Diploma de Estudios Avanzados. Departamento de Psicología Biológica y de la Salud de la, Universidad Autónoma de Madrid.)

- Machado, P. G. B.; Porto-Martins, P. C.; Amorim, C. (2012). Engagement no trabalho entre profissionais da educação.

- Machado, P. G. B. \& Porto-Martins, P. C. (2013). Condições organizacionais enquanto terceiras variáveis entre burnout e engagement. 
Tais dados demonstram, portanto, o estado incipiente que a investigação sobre engagement no trabalho se encontra no Brasil.

\section{REFERÊNCIAS}

ACOSTA, H.; SALANOVA, M.; LLORENS, S. ¿Cómo Predicen las Prácticas Organizacionales el Engagement en el Trabajo en Equipo?: El Rol de la Confianza Organizacional. Ciencia y Trabajo, v. 13, n. 41, p. 125-134, 2013.

AGNST, R.; BENEVIDES-PEREIRA, A. M. T.; PORTO-MARTINS, P. C. (2009). Tradução do manual do UWES Work Engagement Scale. SCHAUFELI, W. B. \& BAKKER, A. B.; 2003. Disponível em <http://www.wilmarschaufeli.nl/ downloads/test-manuals-2/>. Acesso em: 01 fev. 2013.

BAKKER, A. et al. Job resources boost work engagement, particularly when job demands are high. Journal of Educational Psychology, v. 99, n. 2, p. 274-284, 2007.

BAKKER, A. Et al. W. Work engagement: an emerging concept in occupational health psychology. Work \& Stress, v.22, n.3, 187-200, 2008.

BAKKER, A.; LEITER, M. P. Where to go from here: integration and future research on work engagement. In: BAKKER, A. B.; LEITER, M. P. (Org.). Work engagement: a handbook of essential theory and research. New York: Psychology Press, 2010, p.181-196.

BAKKER, A. B.; DEMEROUTI, E.; XANTHOPOULU, D. How do engaged employees stay engaged? Ciencia y Trabajo, v. Special Issue, p. 15-21, 2012.

BAKKER, A.; RODRÍGUEZ-MUÑOZ, A.; DERKS, D. The emergence of positive occupational health psychology. Psicothema, v. 24, n. 1, p. 66-72, 2012.

BENEVIDES-PEREIRA, A. M. T. A saúde mental de profissionais de saúde mental: uma investigação da personalidade de psicólogos. Maringá: EDUEM, 2001.

BENEVIDES-PEREIRA, A. M. T. Burnout: quando o trabalho ameaça o bemestar do trabalhador. São Paulo: Casa do Psicólogo, 2002.

CARROBLES, J. A.; BENEVIDES-PEREIRA, A. M. T. El estrés y la psicología positiva. Em: FERNANDEZ-ABASCAL, E. (Org.). Emociones Positivas. Madrid: Pirámide, 2009.

CARVALHO, V. A. M. L et al. Resiliencia y el modelo Burnout-Engagement en cuidadores formales de ancianos. Psicothema, v. 18, n. 4, p. 791-796, 2006. 
CHIAVENATO, I. Gestão de Pessoas. Rio de Janeiro: Elsevier / Campus, 2008.

CHIAVENATO, I. Recursos Humanos: o capital humanos das organizações. 9. ed. Rio de Janeiro: Elsevier / Campus, 2009.

GIL-MONTE, P. R.; PEIRÓ, J. M. P. Desgaste Psíquico en el Trabajo: el Síndrome de Quemarse. Madrid: Síntesis, 1997.

GIL-MONTE, P. R. El sindrome de quemarse por el trabajo: una enfermedad laboral en la sociedad del bienestar. Madrid: Pirámide, 2005.

GIL-MONTE, P, R.; MORENO-JIMÉNEZ. El sindrome de quemarse por el trabajo (burnout): una perspectiva histórica. Madrid: Pirámide, 2007.

GONZÁLEZ-ROMÁ, V. et al., S. Burnout and work engagement: independent factors or opposite poles? Journal of Vocational Behavior, v. 68, p. 165-174, 2006.

KAHN, W. A. Psychological conditions of personal engagement and disengagement at work. Academy of Management Journal, v. 33, n. 4, p. 692$724,1990$.

KUBOTA, K. et al. Distinción Empírica Entre Engagement y Trabajolismo en Enfermeras Hospitalarias de Japón: Efecto Sobre la Calidad del Sueño y el Desempeño Laboral. Ciencia \& Trabajo, v. 13, n. 41, p. 152-157, 2011.

KUBOTA, K. et al. The Empirical Distinctiveness of Work Engagement and Workaholism among Hospital Nurses in Japan: The effect on Sleep Quality and Job Performance. Ciencia \& Trabajo, v. 14, p. 31-36, 2012.

LEITER, M. P.; MASLACH, C. The impact of interpersonal environment on burnout and organizational commitment. Journal of Organizational Behavior, v. 9, p. 297-308, 1988.

LEITER, M. P. et al. Las Relaciones Interpersonales en el Lugar de Trabajo Como Demandas y Recursos Laborales: Un Modelo de Burnout y Engagement. Ciencia y Trabajo, v. 13, n. 41, p. 143-151, 2011.

LLORENS, S et al. Does a positive gain spiral of resources efficacy beliefs and engagement exist? Computers in Human Behavior, v. 23, p. 825-841, 2007.

MACHADO, P. G. B. Engagement e burnout em motoristas e cobradores de ônibus. V Jornada de Saúde Mental e Psicanálise da PUC-PR: Curitiba, 2010. Disponível em <http://www2.pucpr.br/reol/index.php/jm?dd1=4427\&dd99=view > Acesso em: 23 jun. 2013. 
MACHADO, P. G. B.; BENEVIDES-PEREIRA, A. M. T. Engagement y burnout en profesionales de transporte público urbano. Trabalho de Investigação para a obtenção do Diploma de Estudios Avanzados. Departamento de Psicologia Biológica e da Saúde, Universidade Autónoma de Madrid, 2012.

MACHADO, P. G. B.; PORTO-MARTINS, P. C.; AMORIM, C. Engagement no trabalho entre profissionais da educação. Intersaberes, v. 7, n. 13, p. 193-2014, 2012.

MACHADO, P. G. B.; PORTO-MARTINS, P. C. Condições organizacionais enquanto terceiras variáveis entre burnout e engagement. Diaphora, v. 13, n. 1, p. 35-44, 2013.

MASLACH, C.; GOLDBERG, J. Prevention of burnout: new perspectives. Applied and Preventive Psychology, v. 7, p. 63-74, 1998.

MASLACH, C.; JACKSON, S. E.; LEITER, M. P. Maslach Burnout Inventory Manual, Tercera Edición. Palo Alto CA: Consulting Psychology Press, 1996.

MASLACH, C.; SCHAUFELI, W. B.; LEITER, M. Job burnout. Annual Review of Psychology, v. 52, p. 397-422, 2001.

MASLACH, C.; LEITER, M. P. Early predictors of job burnout and engagement. Journal of Applied Psychology, v. 93, n. 3, p. 498-512, 2008.

MASLOW, A. H. Motivação e personalidade (1954). 2. ed. Nova York: Harper \& Row, 1970.

MORENO-JIMÉNEZ, B. et al. El burnout y el engagement en profesores de Perú: aplicación del modelo de demandas-recursos laborales. Ansiedad y Estrés, v. 16, n. 2-3, p. 293-307, 2010.

OUWENEEL, E.; LE BLANC, P.; SCHAUFELI, W. B. Believe and you will achieve: do changes in self-efficacy correspond with changes in perceived competence, engagement and performance? Applied Psychology: Health and Well-Being, v. 5, p. 225-247, 2013.

PENA, P. G. L., CARDIM, A., ARAÚJO, M. P. N. Taylorismo cibernético e Lesões por Esforços Repetitivos em operadores de telemarketing em SalvadorBahia. Caderno CRH, v. 24, n. 1, p. 133-153, 2011.

POCINHO, M.; PERESTRELO, C. X. Um ensaio sobre burnout, engagement e estratégias de coping na profissão docente. Educação e Pesquisa, v. 37, n. 3, p. 513- 528, 2011. 
PORTO-MARTINS, P. C.; MACHADO, P. G. B. Engagement no trabalho em motoristas e cobradores de ônibus. XL Reunião Anual da Sociedade Brasileira de Psicologia: Curitiba, 2010.

PORTO-MARTINS, P. C.; MACHADO, P. G. B. Engagement en el trabajo: Resenha. Estudos e Pesquisas em Psicologia, v. 10, n. 3, p. 972-976, 2010.

PORTO-MARTINS, P. C.; AMORIM, C. Estresse e engagement no trabalho docente. In Artigo apresentado no X Congresso Nacional de Educação - EDUCERE: Curitiba, PR, 2011.

SALANOVA, M.; SCHAUFELI, W. B.; LLORENS, S; PEIRÓ, J. M \& GRAU, R. Desde el "burnout" al "engagement": ¿una nueva perspectiva? Revista de Psicología del trabajo y de las organizaciones, v. 16, n. 2, p. 117-134, 2000.

SALANOVA, M.; BAKKER, A. B.; LLORENS, S. Flow at work: evidence for an upward spiral of personal and organizational resources. Journal of Happiness Studies, v. 7, p. 1-22, 2006.

SALANOVA, M.; SCHAUFELI, W. B. El engagement en el trabajo. Madrid: Alianza, 2009.

SCHAUFELI, W. B. Work Engagement: what do we know and where do we go? Romanian Journal of Applied Psychology, v. 14, n. 1, p. 3-10, 2012.

SCHAUFELI, W. B et al. The measurement of engagement and burnout: a two sample confirmatory factor analytic approach. Journal of Happiness Studies, v. 3, p. 71-92, 2002.

SCHAUFELI, W. B.; BAKKER, A. Preliminary Manual: Utrecht Work Engagement Scale (UWES). Utrecht: Occupational Health Psychology Unit, 2003.

SCHAUFELI, W. B.; BAKKER, A. B. Job demands, job resources, and their relationship with burnout and engagement: a multi-sample study. Journal of Organizational Behavior, v. 25, p. 293-315, 2004.

SCHAUFELI, W. B.; BAKKER, A. B.; SALANOVA, M. The measurement of work engagement with a short questionnaire: A cross-national study. Educational and Psychological Measurement, v. 66, p. 701-716, 2006.

SCHAUFELI, W.B., BAKKER, A.B.; VAN RHENEN, W. How changes in job demands and resources predict burnout, work engagement, and sickness absenteeism. Journal of Organizational Behavior, v. 30, p. 893-917, 2009. 
SCHAUFELI, W. B.; TARIS, T. W. The Job Demands-Resources model: A critical review. Gedrag \& Organisatie, v. 26, p. 182-204, 2013.

SELIGMAN, M. E. P.; CSIKSZENTMIHALYI, M. Positive psychology an introduction. American Psychologist, v. 55, n. 1, p. 5-14, 2000.

SHIMAZU A.; SCHAUFELI W. B. Is workaholism good or bad for employee wellbeing? The distinctiveness of workaholism and work engagement among Japanese employees. Ind Health., v. 47, p.495-502, 2009.

TETRICK, L. E.; HAIMANN, C. R. Efeitos das demandas ocupacionais, recursos ambientais e pessoais sobre o presenteísmo e saúde. In: ROSSI, A. M.; MEURS, J. A. PERREWÉ (Org.). Stress e qualidade de vida no trabalho (p. 68-85). São Paulo: Atlas, 2013.

TORP, S. et al. Work engagement: a practical measure for workplace health promotion? Health Promotion Int, v. 28, n. 3, p. 387-96, 2012.

Recebido em: 04 de maio de 2011

Aceito em: 29 de agosto de 2013 
